\title{
Who uses self-exclusion to regulate problem gambling? A systematic literature review
}

\author{
FRANZISKA MOTKA ${ }^{1}$, BETTINA GRÜNE ${ }^{1}$, PAWEL SLECZKA ${ }^{2}$, BARBARA BRAUN ${ }^{1}$, JENNY CISNEROS ÖRNBERG ${ }^{3}$ and \\ LUDWIG KRAUS ${ }^{1,3,4 *}$
}

${ }^{1}$ IFT Institut für Therapieforschung, Munich, Germany

${ }^{2}$ Faculty of Health, H:G University for Health and Sport, Ismaning, Germany

${ }^{3}$ Department of Public Health Sciences, Stockholm University, Stockholm, Sweden

${ }^{4}$ Institute of Psychology, ELTE Eötvös Loránd University, Budapest, Hungary

(Received: April 26, 2018; revised manuscript received: August 24, 2018; accepted: August 25, 2018)

\begin{abstract}
Background and aims: Self-exclusion programs offer an intervention for individuals with problem gambling behavior. However, these programs are insufficiently used. This review describes sociodemographic features and gambling behavior of self-excluders as well as goals and motives for initiating self-exclusion from terrestrial and online gambling. In addition, use of further professional help and barriers to self-exclusion are examined. Methods: Based on systematic literature search and quality assessment, $n=16$ original studies (13 quantitative, 2 qualitative, and 1 mixed method) published between 1997 and 2017 in English or German language were analyzed. Results are presented for online and terrestrial gambling separately. Results: Online self-excluders were on average 10 years younger than terrestrial self-excluders. Self-exclusion was mainly motivated by financial problems, followed by feelings of losing control and problems with significant others. Financial problems and significant others were less important for online than for terrestrial gamblers. Main barriers for self-exclusion were complicated enrollment processes, lack of complete exclusion from all venues, little support from venue staff, and lack of adequate information on self-exclusion programs. Both self-excluders from terrestrial and online gambling had negative attitudes toward the need of professional addiction care. Conclusion: To exploit the full potential of self-exclusion as a measure of gambler protection, its acceptance and its utilization need to be increased by target-group-specific information addressing financial issues and the role of significant others, simplifying the administrative processes, facilitating self-exclusion at an early stage of the gambling career, offering self-determined exclusion durations, and promoting additional use of professional addiction care.
\end{abstract}

Keywords: self-exclusion, systematic reviews, regulations, problem gambling, Internet gambling, terrestrial gambling

\section{INTRODUCTION}

Problem and pathological gambling is significantly increasing due to the negative consequences of the affected individual (Gainsbury, 2014), the increasing expansion of the international gambling market (Hayer \& Meyer, 2011b), and the growing use of online gambling (Dragicevic, Percy, Kudic, \& Parke, 2015; Hayer \& Meyer, 2011a). Selfexclusion is considered a particularly restrictive form of gambler protection. Individuals with gambling problems voluntarily exclude themselves from entering one or more gambling venues to prevent further gambling. Gamblers can request an exclusion by, for instance, formally signing an agreement that states a denial of access to all or only particular venues. Moreover, self-exclusion gives gambling providers the opportunity to call gamblers' attention to professional help, such as financial advice, counseling, or other forms of support (Fiedler, 2014; Gainsbury, 2014). There are fundamental differences between features of self-exclusion programs in different countries. These include, for instance, the range of duration of self-exclusion available or whether duration is determined by operators, by law or by gamblers themselves. Besides self-exclusion programs for terrestrial gambling, various gambling platforms provide the opportunity to self-exclude from online gambling on their websites by blocking the account for a specific length of time. Requests for online self-exclusions can be done online using the gamblers' account (for an example, see bwin.com). However, here too, features and principles of online self-exclusion vary between online gambling platforms. While some platforms differ in their selectable length of exclusion (bwin.com and onlinecasino. de/en), other websites do not offer exclusion options at all (palapoker.com).

* Corresponding author: Ludwig Kraus; IFT Institut für Therapieforschung, Leopoldstr. 175, Munich 80804, Germany; Phone: +49 89360804 30; Fax: +49 89360804 19; E-mail: kraus@ift.de

This is an open-access article distributed under the terms of the Creative Commons Attribution-NonCommercial 4.0 International License, which permits unrestricted use, distribution, and reproduction in any medium for non-commercial purposes, provided the original author and source are credited, a link to the CC License is provided, and changes - if any - are indicated. 
Despite great similarities in provided gambling services in terrestrial and online gambling (e.g., slot machines, sports betting, lotteries, roulette, poker, etc.), terrestrial and online gambling differ in some distinctive features. For instance, online gambling is predominantly played at home or on the way using smartphone and is therefore constantly accessible, more anonymous, and more easily concealed from fellows than terrestrial gambling. Moreover, online gambling offers more flexible payment opportunities (Gainsbury, 2015; Gainsbury, Russell, Wood, Hing, \& Blaszczynski, 2015; Gainsbury, Wood, Russell, Hing, \& Blaszczynski, 2012; Griffiths, 2003; Hing, Cherney, et al., 2015). While online and terrestrial gamblers seem to be well researched, little is known about specific differences between self-excluders from terrestrial and online gambling.

Self-exclusion from terrestrial gambling has become an established measure for reducing negative consequences caused by problem gambling behavior in several provinces of Canada and Australia, in some states in the USA and in European countries, such as Germany, Austria, and Switzerland. To date, self-exclusion programs have been evaluated by several studies (e.g., Hayer \& Meyer, 2011b; Hing \& Nuske, 2012; Hing, Tolchard, Nuske, Holdsworth, \& Tiyce, 2014; Ladouceur, Jacques, Giroux, Ferland, \& Leblond, 2000; Ladouceur, Sylvain, \& Gosselin, 2007; Nelson, Kleschinsky, LaBrie, Kaplan, \& Shaffer, 2010; Tremblay, Boutin, \& Ladouceur, 2008), and the results have been summarized in narrative reviews (e.g., Gainsbury, 2014; Kalke, Buth, \& Hayer, 2012; Parke, Parke, Harris, Rigbye, \& Blaszczynski, 2014). The results indicate that self-exclusion has positive effects on gamblers, such as improving financial problems, reducing gambling frequency, and increasing quality of life (Gainsbury, 2014). However, based on the assumption that pathological gamblers need to be protected in order not to harm themselves or significant others, the current state of knowledge suggests that self-exclusion programs are insufficiently used by gamblers and thus do not effectively prevent excessive gambling (Fiedler, 2014; Gainsbury, 2014; O’Neil et al., 2003). For instance, rates of problem gamblers excluded from terrestrial gambling varied between $0.6 \%$ and $17 \%$ in Canada and Australia (Gainsbury, 2014). According to an online survey, the utilization rate of online self-exclusion was $5.4 \%$ and $11 \%$ among all respondents and among respondents classified as at-risk gamblers, respectively (Jonsson, 2008).

Despite growing knowledge concerning the effects and possible benefits of self-exclusion programs, there is a lack of a systematic description of who is using self-exclusion by considering motives as well as barriers for their implementation. Knowledge on particular features of gamblers who exclude themselves from gambling as well as identifying target-groups that are not represented in the group of self-excluders may help improve design and framework of self-exclusion programs as well as enhance acceptance of and access to self-exclusion, and consequently increase its use. In light of growing relevance of online gambling, knowledge on self-exclusion from online gambling and its commonalities as well as differences to self-exclusion from terrestrial gambling may lead to specific strategies promoting the use of self-exclusion within the two domains of terrestrial and online gambling.
The present review aims to address the aforementioned gaps in the current state of research by providing a systematic description of self-excluders of terrestrial and online gambling by focusing (a) on their sociodemographic features, (b) the characteristics of their gambling behavior, and (c) the circumstances surrounding their acceptance of further support. (d) It analyzes their goals and motives as well as (e) barriers for self-exclusion. (f) Differences between selfexcluders from online and terrestrial gambling as well as gender or age group differences and differences between self-excluders and non-self-excluders are reported. Finally, essential characteristics and differences between selfexcluders of terrestrial and online gambling and suggestions for structural changes within existing self-exclusion programs are discussed.

\section{METHODS}

The methodology of this review is based on the proposed framework for systematic reviews by the Centre for Reviews and Dissemination (2009). For quality assessment, the criteria for qualitative and quantitative studies developed by Kmet, Lee, and Cook (2004) were applied. To synthesize the results of the selected studies, the guidelines for a narrative approach were used, recommended by the UK Economic and Social Research Council (Popay et al., 2006).

\section{Search strategy}

A literature search was carried out between 18th and 25th of September 2017 in the following five electronic databases: PsycInfo, PubMed, PSYNDEX, Medline, and Embase. The publication period was set from 1997 to 2017. For the databases PsychInfo and PSYNDEX, the search was limited to studies published in academic journals, while this had to be done manually for the databases PubMed, Medline, and Embase. The search term was composed of two elements: (a) gambling and (b) self-exclusion. For both elements, a list of synonyms and alternative terms were developed, which resulted in gambl* for element (a) and self-exclu*, self exclu*, exclu*, self-ban*, self ban* for element (b). All terms in the list for element (b) were combined with "OR," set in parentheses. The combination of both elements resulted in the search term "gambl* AND (self-exclu* OR self exclu* OR exclu* OR self-ban* OR self ban*)". In total, 911 articles were identified through the database search. After removing duplicates, a total of 420 articles were eligible for screening titles and abstracts. Figure 1 shows the results of the search strategy.

\section{Inclusion and exclusion criteria}

Study selection was conducted independently by the authors FM and BG. Disagreements were resolved through consensus reached by discussion between both authors. Publications were included, if they fulfilled the following general conditions: original study; written in German- or Englishlanguage; using quantitative, qualitative, or mixed methods; and reporting sample size. Relevant studies had to examine a sample of self-excluders, at least as a subgroup, who excluded themselves for terrestrial and/or online gambling. 

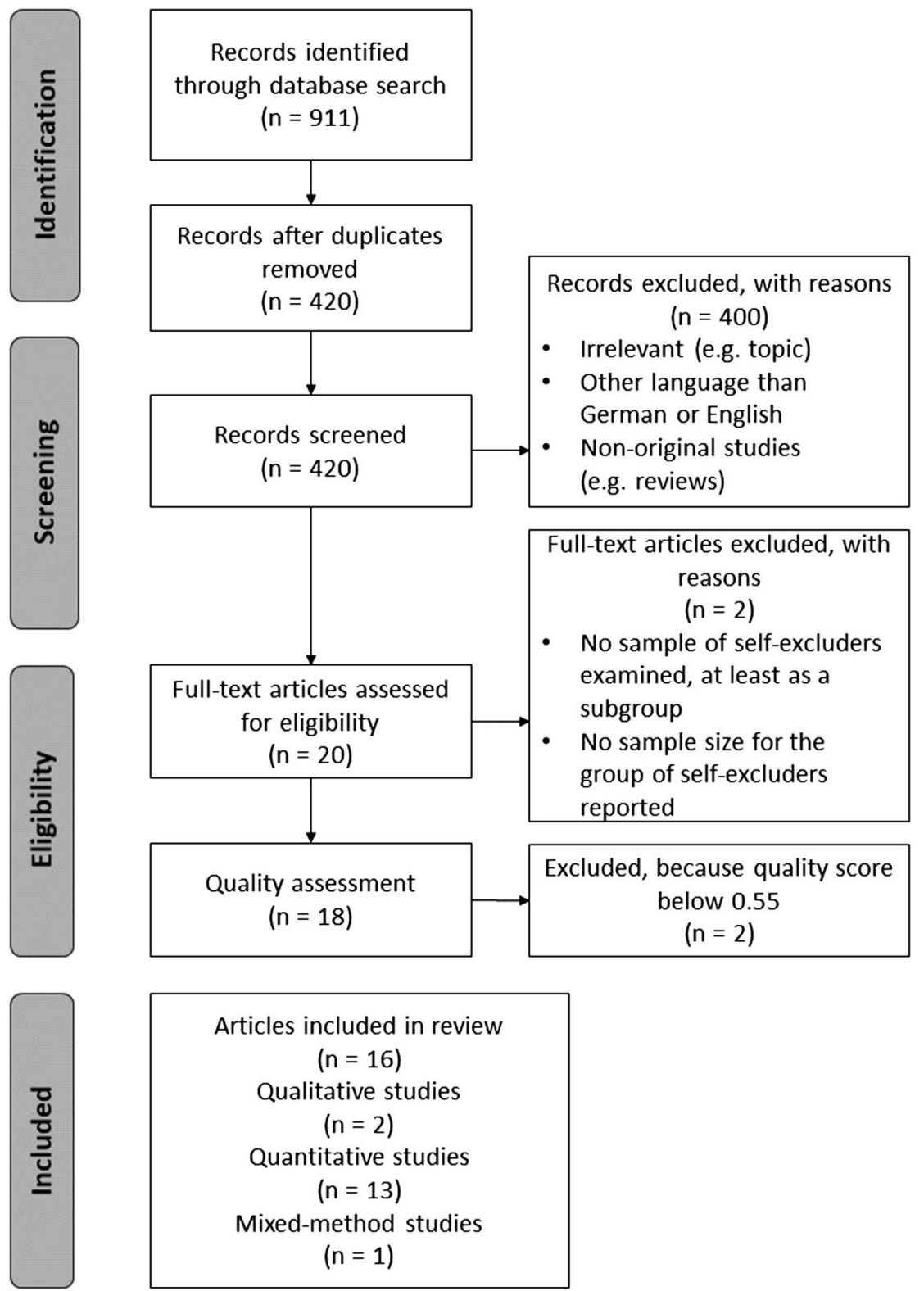

\begin{tabular}{|c|}
\hline Articles included in review \\
$(n=16)$ \\
Qualitative studies \\
$(n=2)$ \\
Quantitative studies \\
$(n=13)$ \\
Mixed-method studies \\
$(n=1)$ \\
\hline
\end{tabular}

Figure 1. PRISMA flow diagram of literature search (Moher, Liberati, Tetzlaff, \& Altman, 2009)

Based on the inclusion and exclusion criteria, a list of 20 articles were generated for a full-text analysis. By analyzing the full-text, two studies were excluded, because they either did not examine self-excluders, at least as a subgroup, or did not report the sample size of examined self-excluders. Figure 1 depicts the flow diagram of the selection process with 18 studies being selected for quality assessment.

\section{Quality assessment}

Due to the variety of methods applied in the identified studies, the authors decided to employ the Standard Quality Assessment Criteria for Evaluating Primary Research Papers from a Variety of Fields (Kmet et al., 2004). This tool allows comprehensive quality scoring using a checklist for qualitative and quantitative studies. For each study, a summary score was calculated by summing the total score and dividing it by the total possible score for each study.
This resulted in 15 articles assessed by the criteria for quantitative studies and three articles assessed by the criteria for qualitative studies. Of the latter ones, one study (Hing \& Nuske, 2012) used a mixed method approach.

FM and BG independently assessed the quality of the included studies. The interrater reliability of the summary scores was excellent, with an interclass correlation coefficient of 0.986 ( $p<.001$; 95\% CI: 0.962, 0.995) (Cicchetti, 1994). For the four differently rated studies, a mean score was calculated. According to the guidelines, studies with a quality score below 0.55 (liberal cut-off point) were excluded $(n=2)$. In total, 16 studies were used for data extraction. All quality scores are displayed in Table 1.

\section{Data extraction and synthesis}

A preliminary synthesis was developed using tabulation, vote count of emergent themes, and thematic analysis. 
Motka et al.

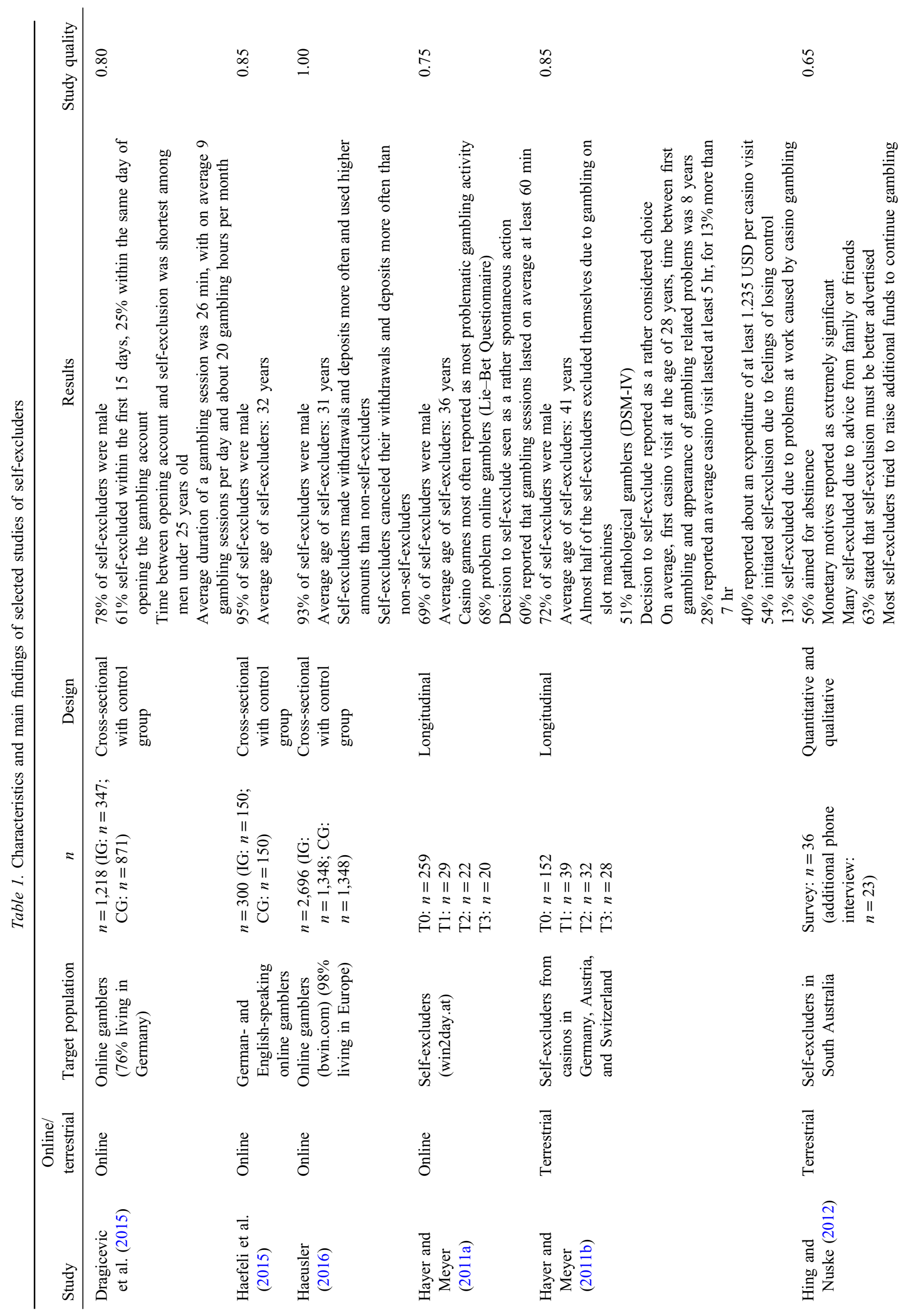


$\stackrel{8}{0}:$

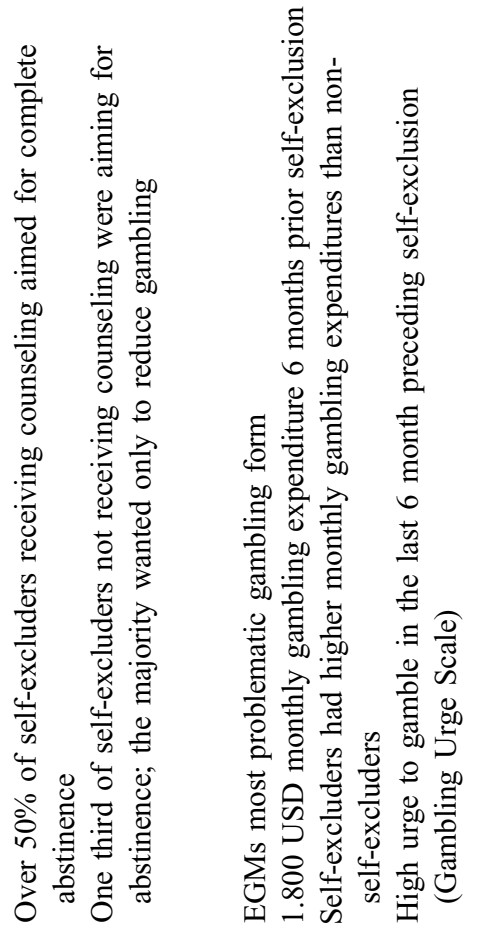

$\stackrel{\infty}{\circ} \stackrel{8}{\circ}$
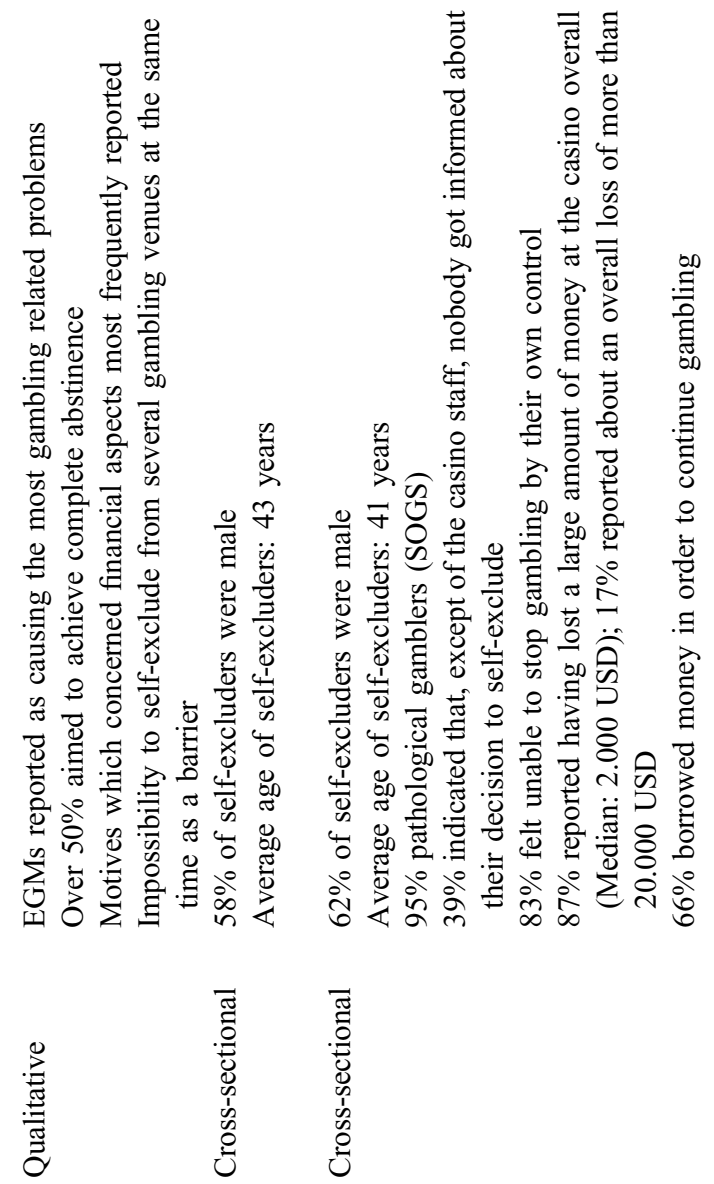

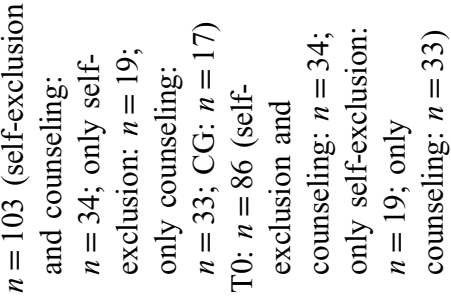<smiles>[CH]CC</smiles>

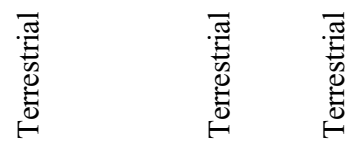

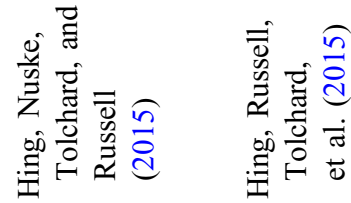


Motka et al.

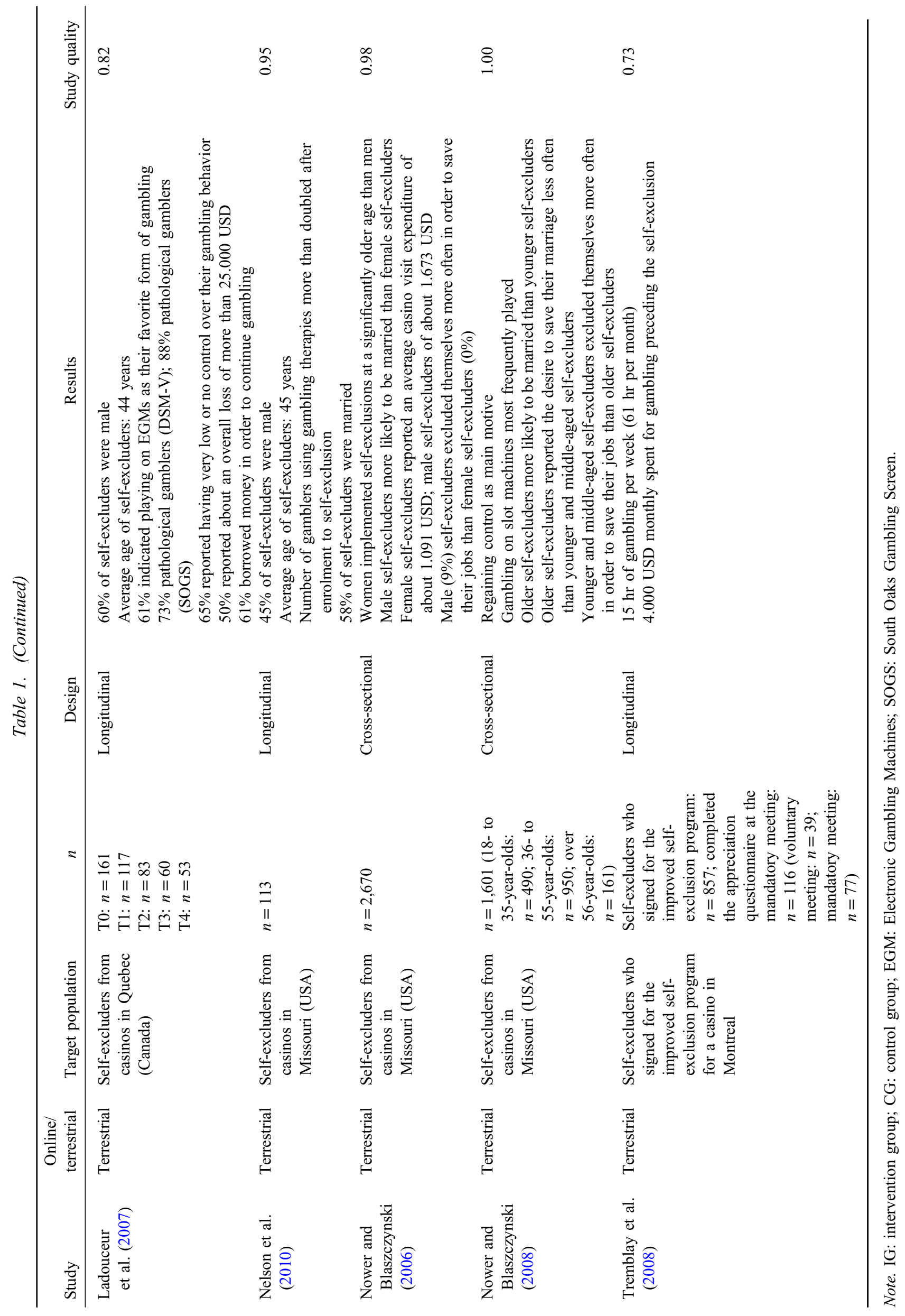


General information, study characteristics, and main results were extracted and tabulated. FM and BG independently conducted the thematic analysis. Relevant primary themes for this review had to be reported in at least five different included studies. In order not to miss out on relevant subthemes, the cut-off level was lowered and subthemes had to be reported in at least four different studies. Details on the identification of themes and subthemes and data extraction are presented in "Supplementary Material." The robustness of the synthesis was ensured through the application of quality assessment (see "Quality assessment" section). In case results were prone to lead to biased synthesis, they were excluded from analyses, for example, prevalence rates and data on demographic characteristics from studies with a sample size lower than 100 self-excluders. Gambling behavior characteristics were only considered when collected prior to or during self-exclusion. This constraint was necessary to ensure that the results did not suffer from a biased implementation of self-exclusion as an intervention.

\section{RESULTS}

\section{Description of studies}

The 16 included studies for data extraction comprised 13 quantitative studies (Dragicevic et al., 2015; Haefeli et al., 2015; Haeusler, 2016; Hayer \& Meyer, 2011a, 2011b; Hing, Russell, Tolchard, et al., 2015; LaBrie et al., 2007; Ladouceur et al., 2000, 2007; Nelson et al., 2010; Nower \& Blaszczynski, 2006, 2008; Tremblay et al., 2008), 2 qualitative studies (Hing, Nuske, et al., 2015; Hing et al., 2014), and 1 mixed method study (Hing \& Nuske, 2012). As shown in Table 1 , the quality scores of all included articles ranged between 0.65 and $1.0(0.0=$ poor quality and $1.0=$ excellent quality). Overall, quantitative studies scored higher (range: $0.73-1.0$ ) than qualitative studies (range: 0.65-0.80). Qualitative studies most often lacked in describing verification procedures to establish credibility/trustworthiness of the study and in outlining the reflexivity within interpretation processes. Of the 16 included studies, 12 examined selfexcluders who self-excluded from terrestrial venues (Hayer \& Meyer, 2011b; Hing \& Nuske, 2012; Hing, Nuske, et al., 2015; Hing, Russell, Tolchard, et al., 2015; Hing et al., 2014; LaBrie et al., 2007; Ladouceur et al., 2000, 2007; Nelson et al., 2010; Nower \& Blaszczynski, 2006, 2008; Tremblay et al., 2008), while four studies examined self-excluders who self-excluded from online gambling websites (Dragicevic et al., 2015; Haefeli et al., 2015; Haeusler, 2016; Hayer \& Meyer, 2011a). None of the qualitative studies examined online self-excluders. Studies that referred to self-exclusion from terrestrial gambling (qualitative and quantitative) surveyed self-excluders from venues in Germany, Austria, and Switzerland $(n=1)$, Queensland (Australia) $(n=3)$, South Australia $(n=1)$, Missouri (USA) $(n=4)$, Quebec (Canada) $(n=2)$, and Montreal (Canada) $(n=1)$. Studies on self-exclusion from online gambling included self-excluders primarily from Europe (Table 1).
For German-speaking countries (Germany, Austria, and Switzerland), only fragmentary information on selfexclusion programs from terrestrial gambling was provided. In Germany, exclusions from state-provided gambling are regulated nationwide by the German State Treaty on Gambling, whereas exclusions from commercially provided gambling are organized by each federal state separately (Motka, Grüne, Braun, \& Kraus, 2018). Switzerland established a country-wide self-exclusion program based on the nationwide data sharing of excluded gamblers. In Austria, gamblers can choose between cross-venue exclusion and the exclusion from specific casinos. In most of the European countries, entry checks are obligatory in order to identify excluded gamblers (Hayer \& Meyer, 2011b). In Queensland (Australia), self-exclusions are implemented for a period of 5 years. Gamblers can choose to be excluded from a venue or from specific gambling activities. Revocations are allowed within $24 \mathrm{hr}$ or after 12 months of enrollment. Patrons and Licensees can be punished for breaches, for example, through financial penalties. Gambling operators have to provide referrals to a gambling counseling service (Hing et al., 2014). Gamblers in South Australia can exclude themselves from one or multiple venues by requesting an interview, either by telephone or by going to an office of the Independent Gambling Authority. During this interview, additional health care support and contacting for followups will be offered (Hing \& Nuske, 2012). In Quebec (Canada), gamblers must approach a security agent who will take them to a private office for enrollment. Gamblers can exclude themselves for at least 6 months; the maximum duration was 5 years in the first study (Ladouceur et al., 2000 ) and 2 years in the second one (Ladouceur et al., 2007). Advertisement for the self-exclusion program is implemented by pamphlets at different areas in casinos. In Montreal (Canada), self-exclusion is implemented within a contact to an information agent. Self-excluders can choose between an exclusion period of 3 months to 5 years. Since 2005 , they can also choose to participate in a regular or an improved self-exclusion program. In the improved program, self-excluders contact a self-exclusion counselor for an evaluation meeting at the beginning and a mandatory meeting at the end of the self-exclusion period. If self-excluders do not attend the mandatory meeting, the self-exclusion period will automatically be continued (Tremblay et al., 2008). In Missouri (USA), gamblers who enroll in the statewide self-exclusion program exclude themselves from any Missouri casino for lifetime (Nelson et al., 2010). The self-exclusion application can be carried out in stateadministered offices or at the venues itself. Self-excluders who breach their exclusion can be arrested or charged with trespassing (Nelson et al., 2010; Nower \& Blaszczynski, 2006, 2008). Information on self-exclusion from online gambling is comparatively rare. In an Austrian gambling website, online gamblers could choose between an exclusion period of $1,3,6$, or 12 months (Hayer \& Meyer, 2011a).

Two studies investigated differences between selfexcluders and groups of gamblers, which used counseling as treatment or no intervention at all (Hing, Nuske, et al., 2015; Hing, Russell, Tolchard, et al., 2015). Other studies compared different subgroups of self-excluders, including 
gender (Nower \& Blaszczynski, 2006), age groups (Nower \& Blaszczynski, 2008), or self-excluders with and without additional use of counseling (Hing, Nuske, et al., 2015; Hing, Russell, Tolchard, et al., 2015). The key parameters and the main findings of the studies included in the review are outlined in Table 1.

\section{Sociodemographic features}

Terrestrial self-excluders. Between $45 \%$ and $72 \%$ of gamblers who excluded themselves from terrestrial gambling were male (Hayer \& Meyer, 2011b; LaBrie et al., 2007; Ladouceur et al., 2000, 2007; Nelson et al., 2010; Nower \& Blaszczynski, 2006). While the gender ratio was largely balanced in American studies (LaBrie et al., 2007; Nelson et al., 2010; Nower \& Blaszczynski, 2006, 2008), the proportion of male self-excluders in German-speaking countries was 72\% (Hayer \& Meyer, 2011b). The average age was between 41 and 45 years (Hayer \& Meyer, 2011b; LaBrie et al., 2007; Ladouceur et al., 2000, 2007; Nelson et al., 2010; Nower \& Blaszczynski, 2006). Between 42\% and $67 \%$ of the self-excluders were married or lived with their spouse or partner (Ladouceur et al., 2000, 2007; Nelson et al., 2010; Nower \& Blaszczynski, 2006, 2008). The majority of self-excluders $(73 \%-90 \%)$ were employed (full-time or part-time) (Ladouceur et al., 2007; Nelson et al., 2010; Nower \& Blaszczynski, 2006, 2008).

Online self-excluders. Between $69 \%$ and $95 \%$ of gamblers who excluded themselves from online platforms were male (Dragicevic et al., 2015; Haefeli et al., 2015; Haeusler, 2016; Hayer \& Meyer, 2011a). The average age ranged between 31 and 36 years (Haefeli et al., 2015; Haeusler, 2016; Hayer \& Meyer, 2011a). Younger online gamblers were more likely to self-exclude than older online gamblers (Dragicevic et al., 2015; Haeusler, 2016). Furthermore, Haeusler (2016) specified the relationship between age and self-exclusion and found a higher likelihood of selfexclusion among gamblers below the average age and among gamblers in the older age groups.

\section{Characteristics of gambling behavior}

Terrestrial self-excluders. Among individuals who selfexcluded from terrestrial gambling, between 51\% and 95\% were classified as (probable) pathological gamblers (Hayer \& Meyer, 2011b; Ladouceur et al., 2000, 2007; Nelson et al., 2010). Based on psychometric scales (Hayer \& Meyer, 2011b; Hing, Russell, Tolchard, et al., 2015) or self-ratings (Hing \& Nuske, 2012; Ladouceur et al., 2007), high levels of urge to gamble prior to self-exclusion were reported. Electronic gambling machines (EGMs), including slot machines, were the favored gambling type, most frequently played and mostly perceived as problematic or seen as the reason for self-exclusion (Hayer \& Meyer, 2011b; Hing \& Nuske, 2012; Hing, Russell, Tolchard, et al., 2015; Hing et al., 2014; Ladouceur et al., 2007; Nower \& Blaszczynski, 2006, 2008). Compared to male self-excluders, female self-excluders started gambling at a later age (Hayer \& Meyer, 2011b; Nower \& Blaszczynski, 2006, 2008).

Studies that examine gambling expenditures of selfexcluders from terrestrial venues reported high spendings prior to self-exclusion. Monthly expenditure ranged on average between 1.800 USD and 4.000 USD (Hing, Russell, Tolchard, et al., 2015; Tremblay et al., 2008). Female selfexcluders' average loss on a casino visit (1.091 USD) was significantly lower than male self-excluders' (1.673 USD) (Nower \& Blaszczynski, 2006). It has also been shown that self-excluders showed higher monthly gambling expenditures than non-self-excluders (Hing, Russell, Tolchard, et al., 2015).

Online self-excluders. Based on the Lie-Bet Questionnaire adapted for Internet gambling, $68 \%$ of online selfexcluders scored above the threshold for problem gambling. Younger self-excluded online gamblers were more often found to fulfill the criteria for problem online gambling than their older counterparts (Hayer \& Meyer, 2011a). According to Dragicevic et al. (2015), online self-excluders most commonly played casino games (e.g., roulette and blackjack), which were also most frequently perceived as problematic gambling activity (Hayer \& Meyer, 2011a).

In the study of Hayer and Meyer (2011a), nearly a quarter of online self-excluders (22\%) reported spending more than 595 USD on gambling per week. Self-excluders made greater monthly losses (962 USD on average) than nonself-excluders (693 USD on average) and also experienced greater variation in monthly losses (Dragicevic et al., 2015). Self-excluded online gamblers also made higher and more frequently withdrawals and deposits and also canceled them more often than non-self-excluders (Haeusler, 2016).

\section{Additional health care support}

Terrestrial self-excluders. Across studies, utilization of additional health care support was diverse. Studies surveying the prevalence rate during enrollment to self-exclusion reported that around $10 \%$ of self-excluders had any previous or current experience of individual professional support (Hayer \& Meyer, 2011b; Ladouceur et al., 2000). Longitudinal studies surveying the prevalence rate after a few months of self-exclusion found percentages around 20\%-30\% (Hayer \& Meyer, 2011b; Nelson et al., 2010; Nower \& Blaszczynski, 2006). In line with this, Nelson et al. (2010) reported that the number of gamblers utilizing gambling therapies more than doubled after self-exclusion. There was no significant difference in seeking gambling counseling between females and males (Nower \& Blaszczysnki, 2006). Furthermore, professional help was more often used by gamblers with the primary goal of abstinence, a higher problem severity, as well as specific psychological features, such as lower levels of independence and pride in resolving problems (Hing, Nuske, et al., 2015).

Studies showed inconsistencies in the attitude toward the combination of self-exclusion and health care support among self-excluders (Hayer \& Meyer, 2011b; Hing \& Nuske, 2012; Hing, Nuske, et al., 2015). For instance, Hayer and Meyer (2011b) reported an overall disagreement among self-excluders to the necessity to combine selfexclusion with counseling (Hayer \& Meyer, 2011b). Hing, Nuske, et al. (2015) found that the opinion about additional use of counseling varied according to treatment status. Selfexcluders without additional use of counseling expressed more negative attitudes toward counseling. 
Online self-excluders. The prevalence of additional health care support of online self-excluders was solely surveyed by Hayer and Meyer (2011a). About 1\% of self-excluders reported that they decided to exclude as part of their gambling counseling. Similar to terrestrial selfexcluders, online self-excluders strongly disagreed with the necessity to combine self-exclusion with counseling or therapy.

\section{Goals and motives}

Terrestrial self-excluders. Achieving abstinence was the main goal behind self-exclusion for the majority of gamblers, and reducing gambling behavior was rated second (Hing \& Nuske, 2012; Hing et al., 2014). However, goals differed by treatment status. While for the majority of selfexcluders who additionally made use of counseling services, the main goal was to reach abstinence, the majority of selfexcluders without using counseling services wanted to reduce gambling by restricting access to their regularly visited venues (Hing, Nuske, et al., 2015).

Most self-excluders involved others (e.g., counselor, family, or friends) in their decision to self-exclude. Only a small percentage reported that nobody else knew about their selfexclusion (Hing \& Nuske, 2012; Ladouceur et al., 2000). The involvement of others varied by treatment status. While only a small proportion of self-excluders without use of counseling made their decision along with others, the majority of self-excluders who made additional use of counseling involved people close to them (Hing, Nuske, et al., 2015; Hing et al., 2014). Gamblers using selfexclusion and counseling generally considered their counselor important for their decision to self-exclude (Hayer \& Meyer, 2011b; Hing \& Nuske, 2012, Hing, Nuske, et al., 2015; Hing et al., 2014; Nower \& Blaszczynski, 2006).

The most important or most frequently named reasons for self-exclusion were financial motives (Hing \& Nuske, 2012; Hing et al., 2014; Ladouceur et al., 2007). According to Hayer and Meyer (2011b), 76\% of the self-excluders stated that they had lost too much money, $44 \%$ reported financial problems due to casino gambling, $37 \%$ mentioned to have placed bets that were in no relation to their income level or wealth, and $29 \%$ reported to be in debts because of their casino gambling. A further frequently named motive for self-exclusion was regaining control (Hayer \& Meyer, 2011b, Nelson et al., 2010; Nower \& Blaszczynski, 2008). Women (55\%) slightly more often than men (49\%) mentioned the wish to gain control as motive for self-exclusion (Nower \& Blaszczynski, 2006).

Significant others (e.g., family members or friends) played a key role in the decision for self-exclusion (Hing \& Nuske, 2012; Hing, Nuske, et al., 2015; Hing et al., 2014). Family members or the partner were considered more important for the decision to self-exclude than friends and colleagues (Hing \& Nuske, 2012). About 20\% of selfexcluders initiated to be banned on the advice, request, or pressure of their partners, family members, or friends (Hayer \& Meyer, 2011b; Ladouceur et al., 2000; Nelson et al., 2010; Nower \& Blaszczynski, 2006). One third of self-excluders reported that they self-excluded due to family or relationship problems caused by casino gambling
(Hayer \& Meyer, 2011b). In line with this, the wish to protect their marriage was named by about $20 \%$ of selfexcluders (Nower \& Blaszczynski, 2006).

Health and psychological problems of self-excluders were frequently reported as important reason for selfexclusion (Hing \& Nuske, 2012; Hing et al., 2014). Women as well as older self-excluders were more likely to report prevention of suicide as motive for self-exclusion than their respective counterparts (Nower \& Blaszczynski, 2006, 2008). Motives related to work were rated as rather unimportant (Hayer \& Meyer, 2011b; Hing \& Nuske, 2012; Nower \& Blaszczynski, 2006, 2008).

Online self-excluders. Only one study surveyed goals and reasons for self-exclusion of online self-excluders (Hayer \& Meyer 2011a). About one quarter (24\%) of online self-excluders wanted to abstain from all forms of gambling. Nearly the same proportion (22\%) wanted to abstain only from online gambling and $13 \%$ only wanted to stop gambling on a specific gambling platform. The most commonly named reasons for self-exclusion were financial problems. About half (52\%) of the online self-excluders mentioned an excessive loss of money, about one quarter (26\%) placing bets that were in no relation to their income level or wealth and almost one fifth (19\%) reported general financial problems. Debts were a decisive motive for $12 \%$ and loss of control for $30 \%$ of online self-excluded gamblers. Motives related to the gambler's social environment were reported comparatively rarely. Merely $15 \%$ stated that they had excluded themselves due to family or relationship problems and $5 \%$ on the advice of family or friends. Problems at work were reported by $3 \%$ as motive for self-exclusion (Hayer \& Meyer, 2011a).

\section{Barriers for self-exclusion}

Barriers for self-exclusion were only investigated among terrestrial gamblers. The self-exclusion enrollment was commonly seen as time-consuming and embarrassing, especially when simultaneous exclusion from multiple venues was impossible. During the process of enrollment, privacy and confidentiality were an important concern (Hing, Nuske, et al., 2015; Hing et al., 2014). Venue staffs' attitude was also frequently criticized: staff members were perceived as not sufficiently briefed on the process and did not provide reasonable sensitivity, encouragement, or support. Selfexcluders also reported that they were not given adequate information on the self-exclusion program (e.g., penalties for breaches and additional counseling services) (Hing \& Nuske, 2012; Hing et al., 2014; Nelson et al., 2010). Moreover, self-excluders were not confident about the detection of breaches against exclusion (Hing \& Nuske, 2012; Hing, Nuske, et al., 2015; Hing et al., 2014; Ladouceur et al., 2000). Frequently, it was also criticized that self-exclusion programs were not sufficiently advertised (Hing \& Nuske, 2012; Hing et al., 2014; Ladouceur et al., 2000).

The impossibility of excluding from several venues at the same time, resulting in opportunities for gambling at alternative venues, was negatively evaluated in studies without the possibility of simultaneous multivenue exclusion (Hing, Nuske, et al., 2015; Hing et al., 2014). Self-excluders 
without using additional counseling were less concerned about excluding individually from multiple venues due to their primary goal of reducing their gambling behavior (Hing, Nuske, et al., 2015). Opinions on the length of the ban varied with some self-excluders preferring short time and others preferring lifetime ban (Hing et al., 2014; Nelson et al., 2010). Self-excluders in a Canadian study criticized the absence of a follow-up process during self-exclusion and the lack of additional clinical support. They were also critical about the organization of renewing exclusion, which had to be made personally at the venue (Ladouceur et al., 2000).

\section{DISCUSSION}

Our review provides the first systematic description of individuals who have self-excluded from terrestrial and online gambling. The description included basic sociodemographic features, gambling behavior characteristics, the use of additional help, goals, and motives as well as barriers for self-exclusion.

With regard to sociodemographic characteristics, individuals who self-excluded from terrestrial gambling were generally men in their early to mid-40s. Gamblers who had excluded themselves from online gambling were on average 10 years younger than self-excluders from terrestrial gambling. These results are in line with studies showing a higher prevalence of problem gambling among men compared to women as well as a younger average age of online gamblers compared to terrestrial gamblers (Dowling et al., 2017; Johansson, Grant, Kim, Odlaug, \& Götestam, 2009; Hing, Russell, Gainsbury, \& Blaszczynski, 2015; Wardle, Moody, Griffiths, Orford, \& Volberg, 2011; Wood \& Williams, 2011). Moreover, the results show an uneven gender ratio between self-excluders from terrestrial and online gambling with a higher proportion of men among self-excluders from online gambling than from terrestrial gambling. This observation is consistent with the results of previous studies (Blaszczynski, Russell, Gainsbury, \& Hing, 2016; Gainsbury et al., 2012; Hing, Russell, Gainsbury, et al., 2015).

Gambling on EGMs, including slot machines, was identified as the form of terrestrial gambling that most frequently led to the development of gambling-related problems among self-excluded gamblers. This confirms observations of studies reporting EGMs and slot machines as high-risk games more strongly associated with gambling problems than other types of gambling (Breen \& Zimmerman, 2002; Hodgins et al., 2012; Lund, 2006; Meyer, Häfeli, Mörsen, \& Fiebig, 2010; Petry, 2003). Therefore, self-exclusion programs should be tailored to the high-risk group of EGM and slot machine gamblers.

The major motives for initiating self-exclusion were financial issues and significant others. While for gamblers self-excluded from terrestrial gambling, significant others played an important role in decision-making; they had only little impact on gamblers self-excluded from online gambling. This might be related to findings that online gamblers were less often married or living with a partner than gamblers of terrestrial gambling (Griffiths, Wardle, Orford, Sproston, \& Erens, 2009; Wood \& Williams, 2009).
Despite potentially problematic features specific for online gambling, for example, high accessibility and anonymity as well as the use of immature payments (Griffiths, 2003; Siemens \& Kopp, 2011), gamblers excluded from online gambling reported financial reasons less often than gamblers excluded from terrestrial gambling. This may be explained by sociodemographic differences between online and terrestrial gamblers. Results suggest that online gamblers have a higher sociodemographic status, a higher household income and are more likely to work full-time than terrestrial gamblers (Gainsbury et al., 2012; Griffiths et al., 2009; Wood \& Williams, 2011). It may alternatively relate to the younger age of self-excluded online gamblers and the fewer financial obligations related to this, that is, self-excluded online gamblers are presumably less likely to have started a family. Moreover, online gamblers may have made use of self-exclusion earlier than terrestrial gamblers due to online self-exclusion being more anonymous and faster to implement. Early self-exclusion might prevent (at least in part) financial problems, and hence, they may have been less important for self-excluders from online gambling. These findings illustrate that advertisement and media campaigns for self-exclusion should be tailored to the specific motives and needs of online and terrestrial gamblers.

The primary aim of self-exclusion was to prevent further gambling and to facilitate abstinence (Hayer \& Meyer, 2011a, 2011b; Hing \& Nuske, 2012; Hing et al., 2014). This is not surprising considering the fact that the majority of self-excluders from terrestrial or online gambling were identified to gamble problematically or pathologically. This illustrates that the concept of self-exclusion meets the needs of problem and pathological gamblers to regain control over their gambling behavior. However, these results also underline that self-exclusion seems to be used more often as a measure to regulate gambling behavior and to reduce further negative consequences at a later stage of the problem gambling career rather than a measure of early intervention. This supports previous notions of self-exclusion as a measure of harm minimization (Gainsbury, 2014; Hayer \& Meyer, 2011b; Hing \& Nuske, 2012; Hing, Russell, Gainsbury, et al., 2015; Hing et al., 2014). Nevertheless, if initiated earlier, self-exclusion may also be used to prevent intensive forms of gambling and consequently the development of problem and pathological gambling. To increase the effectiveness of self-exclusion and to promote early utilization, self-exclusion needs to be promoted and advertised as an effective means to regulate gambling behavior. In addition, self-exclusion programs for terrestrial gambling as well as self-exclusion options for online gambling need major structural changes to meet the needs of gamblers who wish to take a break from gambling as early intervention measure.

The necessity to improve exclusion programs for terrestrial gambling was underlined by the evaluation of frequently named structural barriers to self-exclusion that delay or prevent gamblers from using self-exclusion, such as impossibility of multiple venue exclusion and problems with venue staff due to false or incomplete information or lack of sensitivity and privacy (Gainsbury, 2014; Hing, Nuske, et al., 2015; Hing et al., 2014; Parke et al., 2014). Availability and accessibility are the key factors that should be considered in the (re)organization of 
self-exclusion. For instance, (a) gamblers should be able to exclude themselves from multiple venues within a single enrollment preferably outside of gambling venues. In addition, self-exclusion programs should be organized state-wide. Multiple venue exclusion reduces shame and the exposure to gambling within enrollment procedures. Moreover, restricting access to multiple venues will increase the effectiveness of self-exclusion by reducing the likelihood of circumvention and prevention of further gambling; (b) gamblers should be able to determine the length of selfexclusion themselves (Gainsbury, 2014). This might also encourage gamblers at an early stage of their problem gambling career to initiate self-exclusion and to experience a time out from gambling; (c) venue staff members need to be trained in all aspects of self-exclusion, for example, giving adequate information, treating applicants respectfully, ensuring adherence to self-exclusion by strict identity checks, etc. Moreover, staff members need to be trained in early recognition of problem gambling behavior and they should be encouraged to motivate possible problem gamblers to take a break from gambling. By improving staffs' competences and adherence to rules, gamblers will establish trust in self-exclusion programs.

Although research on barriers to self-exclusion from online gambling is lacking, the results indicate rather diverse abstinence goals. To address different needs, gambling providers should enable self-excluders to make individual decisions on the duration as well as on the extent of the exclusion, for example, exclusion from specific types of gambling or blocking the account. While some gambling providers already offer different self-exclusion options, this should be mandatory for all gambling websites. However, the exclusion is then limited to a single provider and therefore legal regulations and technical solutions are necessary to prevent further gambling using another gambling website or creating a new account.

The results indicate that the utilization of additional counseling increased during the self-exclusion period. However, additional forms of support were utilized rather rarely. In general, the use of further health care offers strongly depends on the interconnection between selfexclusion programs and further intervention measures. For example, accepting a direct offer of further professional help within the process of self-exclusion needs less self-initiative and is therefore more likely than active help seeking by the gambler. Besides offers for additional professional help at enrollment, follow-ups should be a tool accompanying selfexclusion. By this, further professional help can be offered through the whole self-exclusion period and help to increase their utilization. The self-exclusion program in Montreal (Canada) can be considered as a good example of the interconnection of self-exclusion and other forms of support. Here, gamblers are offered a session with an independent psychological adviser during the implementation of self-exclusion, who provides information on options for professional help (Tremblay et al., 2008). In contrast, the links between the German self-exclusion program and other offers of support have been evaluated as not sufficiently developed (Fiedler, 2014).

When interpreting the results of this review, some limitations need to be considered. First, the included studies used different methodological approaches, for example, quantitative and qualitative. Moreover, quantitative data are based on self-reports, self-exclusion files, or behavioral tracking data. With regard to the aims of this review, the use of this broad range of methodological approaches was beneficial, especially concerning the analyses of aspects that were not constantly focused in research to date, for example, motives or perceived barriers related to self-exclusion. Second, selfexclusion of gamblers is a rather new research field, where randomized controlled trials or other more rigorous methods are practically impossible or difficult to perform. Our results were thus extracted from mostly descriptive studies, which ranged in their quality. To ensure the highest possible data quality, we only included peer-reviewed publications and assessed their data quality. Publications below the recommended thresholds were excluded. Third, the results may be biased by the different self-exclusion programs in countries included in the study. Due to the scarce information given in the studies on self-exclusion jurisdictions and regulations, generalizability of results and conclusions may be limited or only applicable to certain programs. Finally, due to lack of data, cultural factors that may influence gamblers' attitudes toward the use of self-exclusion could not be considered.

\section{CONCLUSIONS}

Our review replicated some results and implications of previous narrative reviews, but in addition, brought new insights, especially with regard to online self-exclusion and the unused potential of self-exclusion as early intervention measure. To exploit the full potential of self-exclusion as a measure of gambler protection, its acceptance and its utilization need to be increased. First, self-exclusion programs for terrestrial gambling and online self-exclusion options as well as their promotion need to be target-group-specific and should be tailored to high-risk groups. Moreover, the promotion activities and information material should address motives for self-exclusion, such as financial issues or the role of significant others, especially in terms of terrestrial gambling. Second, the potential of self-exclusion as an early intervention measure is not fully exploited. To facilitate the use of self-exclusion as a time-out option at an early stage of problematic gambling behavior, venue staff needs to be trained in their skills of early recognition of problem gambling. In terms of online gambling, pop-up messages should inform problem gamblers about self-exclusion and additional care. Third, barriers to self-exclusion need to be reduced. To encourage early utilization, the process should be simplified, for instance, by providing options to selfexclude outside of gambling venues and to self-exclude from multiple venues within one enrollment procedure. Most importantly, the duration of self-exclusion should be more flexible to meet the needs of terrestrial and online gamblers. This includes individual decisions on the duration of self-exclusion. Fourth, for self-exclusion to become a measure of regulating impaired impulse control rather than merely controlling gambling exposure, self-exclusion and professional addiction care need to be strongly connected.

Finally, some future research directions became apparent. In light of the growing relevance of online gambling, 
it is necessary to shed light on the mechanisms behind the decision to self-exclude, for example, with regard to barriers. Moreover, knowledge concerning early identification on the basis of behavioral data is favorable for both offline and online gambling. Concerning the length of the exclusion period, studies investigating the impact of self-determined duration on its use and future gambling behavior are highly recommended.

Funding sources: FM, BG, PS, BB, and LK were supported by the Bavarian State Ministry of Finance, Regional Development and Regional Identity via the Bavarian State Ministry of Public Health and Care Services in the context of the Bavarian Coordination Centre for Gambling Issues (LSG Bayern). Funding for JCÖ and LK was provided within the frame of the Swedish program grant "Responding to and Reducing Gambling Problems - Studies in Help-seeking, Measurement, Comorbidity and Policy Impacts" financed by the Swedish Research Council for Health, Working Life and Welfare (Forte), grant number 2016-07091.

Authors' contribution: FM and BG implemented the methodical steps for the systematic research process and wrote the first draft of the manuscript. All authors developed the research questions, gave feedback for the manuscript, had full access to all data, take responsibility for the integrity of the data, and have approved the final version of the manuscript.

Conflict of interest: The State of Bavaria provides gambling services (lotteries, sports betting, and casino games) within the state gambling monopoly via the State Lottery Administration and provides funding to the Bavarian Coordination Centre for Gambling Issues (LSG Bayern) as an unrestricted grant. The authors have no further conflict of interest to declare.

\section{REFERENCES}

Blaszczynski, A., Russell, A., Gainsbury, S., \& Hing, N. (2016). Mental health and online, land-based and mixed gamblers. Journal of Gambling Studies, 32(1), 261-275. doi:10.1007/ s10899-015-9528-Z

Breen, R. B., \& Zimmerman, M. (2002). Rapid onset of pathological gambling in machine gamblers. Journal of Gambling Studies, 18(1), 31-43. doi:10.1023/A:1014580112648

Centre for Reviews and Dissemination. (2009). Systematic reviews: CRD's guidance for undertaking reviews in health care. York: CRD, University of York. Retrieved from https:// www.york.ac.uk/media/crd/Systematic_Reviews.pdf

Cicchetti, D. V. (1994). Guidelines, criteria, and rules of thumb for evaluating normed and standardized assessment instruments in psychology. Psychological Assessment, 6(4), 284-290. doi:10.1037/1040-3590.6.4.284

Dowling, N. A., Merkouris, S. S., Greenwood, C. J., Oldenhof, E., Toumbourou, J. W., \& Youssef, G. J. (2017). Early risk and protective factors for problem gambling: A systematic review and meta-analysis of longitudinal studies. Clinical Psychology Review, 51, 109-124. doi:10.1016/j.cpr.2016.10.008

Dragicevic, S., Percy, C., Kudic, A., \& Parke, J. (2015). A descriptive analysis of demographic and behavioral data from Internet gamblers and those who self-exclude from online gambling platforms. Journal of Gambling Studies, 31(1), 105-132. doi:10.1007/s10899-013-9418-1

Fiedler, I. (2014). Evaluierung des Sperrsystems in deutschen Spielbanken [Evaluation of the exclusion system in German casinos]. Hamburg, Germany: Universität Hamburg. Retrieved from https://www.bwl.uni-hamburg.de/irdw/dokumente/publikationen/ evaluierung-von-sperrsystemen-in-spielbanken.pdf

Gainsbury, S. M. (2014). Review of self-exclusion from gambling venues as an intervention for problem gambling. Journal of Gambling Studies, 30(2), 229-251. doi:10.1007/s10899-0139362-0

Gainsbury, S. M. (2015). Online gambling addiction: The relationship between Internet gambling and disordered gambling. Current Addiction Reports, 2(2), 185-193. doi:10.1007/ s40429-015-0057-8

Gainsbury, S. M., Russell, A., Wood, R., Hing, N., \& Blaszczynski, A. (2015). How risky is Internet gambling? A comparison of subgroups of internet gamblers based on problem gambling status. New Media \& Society, 17(6), 861-879. doi:10.1177/1461444813518185

Gainsbury, S. M., Wood, R., Russell, A., Hing, N., \& Blaszczynski, A. (2012). A digital revolution: Comparison of demographic profiles, attitudes and gambling behavior of Internet and non-Internet gamblers. Computers in Human Behavior, 28(4), 1388-1398. doi:10.1016/j.chb.2012.02.024

Griffiths, M. (2003). Internet gambling: Issues, concerns, and recommendations. CyberPsychology \& Behavior, 6(6), 557568. doi:10.1089/109493103322725333

Griffiths, M., Wardle, H., Orford, J., Sproston, K., \& Erens, B. (2009). Sociodemographic correlates of Internet gambling: Findings from the 2007 British Gambling Prevalence Survey. CyberPsychology \& Behavior, 12(2), 199-202. doi:10.1089/ cpb.2008.0196

Haefeli, J., Lischer, S., \& Haeusler, J. (2015). Communicationsbased early detection of gambling-related problems in online gambling. International Gambling Studies, 15(1), 23-38. doi:10.1080/14459795.2014.980297

Haeusler, J. (2016). Follow the money: Using payment behavior as predictor for future self-exclusion. International Gambling Studies, 16(2), 246-262. doi:10.1080/14459795.2016.1158306

Hayer, T., \& Meyer, G. (2011a). Internet self-exclusion: Characteristics of self-excluded gamblers and preliminary evidence for its effectiveness. International Journal of Mental Health and Addiction, 9(3), 296-307. doi:10.1007/s11469-010-9288-z

Hayer, T., \& Meyer, G. (2011b). Self-exclusion as a harm minimization strategy: Evidence for the casino sector from selected European countries. Journal of Gambling Studies, 27(4), 685-700. doi:10.1007/s10899-010-9227-8

Hing, N., Cherney, L., Gainsbury, S. M., Lubman, D. I., Wood, R. T., \& Blaszczynski, A. (2015). Maintaining and losing control during Internet gambling: A qualitative study of gamblers' experiences. New Media \& Society, 17(7), 1075-1095. doi:10.1177/1461444814521140

Hing, N., \& Nuske, E. (2012). The self-exclusion experience for problem gamblers in South Australia. Australian Social Work, 65(4), 457-473. doi:10.1080/0312407X.2011.594955 
Hing, N., Nuske, E., Tolchard, B., \& Russell, A. (2015). What influences the types of help that problem gamblers choose? A preliminary grounded theory model. International Journal of Mental Health Addiction, 13(2), 241-256. doi:10.1007/ s11469-014-9525-y

Hing, N., Russell, A. M. T., Gainsbury, S. M., \& Blaszczynski, A. (2015). Characteristics and help-seeking behaviors of Internet gamblers based on most problematic mode of gambling. Journal of Medical Internet Research, 17(1), e13. doi:10.2196/jmir.3781

Hing, N., Russell, A., Tolchard, B., \& Nuske, E. (2015). Are there distinctive outcomes from self-exclusion? An exploratory study comparing gamblers who have self-excluded, received counselling, or both. International Journal of Mental Health and Addiction, 13(4), 481-496. doi:10.1007/s11469-015-9554-1

Hing, N., Tolchard, B., Nuske, E., Holdsworth, L., \& Tiyce, M. (2014). A process evaluation of a self-exclusion program: A qualitative investigation from the perspective of excluders and non-excluders. International Journal of Mental Health and Addiction, 12(4), 509-523. doi:10.1007/s11469-014-9482-5

Hodgins, D. C., Schopflocher, D. P., Martin, C. R., el-Guebaly, N., Casey, D. M., Currie, S. R., Smith, G. J., \& Williams, R. J. (2012). Disordered gambling among higher-frequency gamblers: Who is at risk? Psychological Medicine, 42(11), 2433-2444. doi:10.1017/S0033291712000724

Johansson, A., Grant, J. E., Kim, S. W., Odlaug, B. L., \& Götestam, K. G. (2009). Risk factors for problematic gambling: A critical literature review. Journal of Gambling Studies, 25(1), 67-92. doi:10.1007/s10899-008-9088-6

Jonsson, J. (2008). Responsible gaming and gambling problems among 3,000 Swedish Internet poker players. Paper presented at the 7th European Conference on Gambling Studies and Policy Issues, Nova Gorica, Slovenia. Retrieved from https:// www.easg.org/media/file/conferences/novagorica2008/thursday/ 1400-ses3/jonsson_jakob.pdf

Kalke, J., Buth, S., \& Hayer, T. (2012). Indizierte Prävention im Glücksspielbereich. Wissenschaftlicher Kenntnisstand und zukünftige Herausforderungen [Induced prevention in the gambling field. Scientific level of knowledge und prospective challenges]. Sucht, 58(6), 359-368. doi:10.1024/0939-5911. a000213

Kmet, L. M., Lee, R. C., \& Cook, L. S. (2004). Standard quality assessment criteria for evaluating primary research papers from a variety of fields. Edmonton, AB: Alberta Heritage Foundation for Medical Research. Retrieved from https:// www.ihe.ca/advanced-search/standard-quality-assessmentcriteria-for-evaluating-primary-research-papers-from-a-varietyof-fields

LaBrie, R. A., Nelson, S. E., LaPlante, D. A., Peller, A. J., Caro, G., \& Shaffer, H. J. (2007). Missouri casino self-excluders: Distributions across time and space. Journal of Gambling Studies, 23(2), 231-243. doi:10.1007/s10899-006-9037-1

Ladouceur, R., Jacques, C., Giroux, I., Ferland, F., \& Leblond, J. (2000). Analysis of a casino's self-exclusion program. Journal of Gambling Studies, 16(4), 453-460. doi:10.1023/ A:1009488308348

Ladouceur, R., Sylvain, C., \& Gosselin, P. (2007). Self-exclusion program: A longitudinal evaluation study. Journal of Gambling Studies, 23(1), 85-94. doi:10.1007/s10899-006-9032-6

Lund, I. (2006). Gambling and problem gambling in Norway: What part does the gambling machine play? Addiction
Research and Theory, 14(5), 475-491. doi:10.1080/ 16066350600836825

Meyer, G., Häfeli, J., Mörsen, C. P., \& Fiebig, M. (2010). Die Einschätzung des Gefährdungspotentials von Glücksspielen [The evaluation of the risk potential of gambling]. Sucht, 56(6), 405-414. doi:10.1024/0939-5911/a000057

Moher, D., Liberati, A., Tetzlaff, J., \& Altman, D. G. (2009). Preferred reporting items for systematic reviews and metaanalyses: The PRISMA statement. PLOS Medicine, 6(7), e1000097. doi:10.1371/journal.pmed.1000097

Motka, F., Grüne, B., Braun, B., \& Kraus, L. (2018). Spielersperren in Deutschland: Stand der gesetzlichen Glücksspielregelungen und ihre Umsetzung [Exclusion of gambling in Germany: Legal regulations and implementation]. Suchttherapie. Advance online Publication. doi:10.1055/ a-0583-2888

Nelson, S. E., Kleschinsky, J. H., LaBrie, R. A., Kaplan, S., \& Shaffer, H. J. (2010). One decade of self exclusion: Missouri casino self-excluders four to ten years after enrollment. Journal of Gambling Studies, 26(1), 129-144. doi:10.1007/s10899009-9157-5

Nower, L., \& Blaszczynski, A. (2006). Characteristics and gender differences among self-excluded casino problem gamblers: Missouri data. Journal of Gambling Studies, 22(1), 81-99. doi:10.1007/s10899-005-9004-2

Nower, L., \& Blaszczynski, A. (2008). Characteristics of problem gamblers 56 years of age or older: A statewide study of casino self-excluders. Psychology and Aging, 23(3), 577-584. doi: $10.1037 / \mathrm{a} 0013233$

O’Neil, M., Whetton, S., Dolman, B., Herbert, M., Giannopoulus, V., O'Neil, D., \& Wordley, J. (2003). Evaluation of selfexclusion programs and harm minimisation measures - Report A. Victoria, Australia: Gambling Research Panel. Retrieved from https://www.adelaide.edu.au/saces/docs/publicationseports/completereportselfexclusiona.pdf

Parke, J., Parke, A., Harris, A., Rigbye, J., \& Blaszczynski, A. (2014). Restricting access: Self-exclusion as a gambling harm minimisation measure in Great Britain. The Journal of Gambling Business and Economics, 8, 52-92. Retrieved from http:// www.bjll.org/index.php/jgbe/article/view/1032

Petry, N. M. (2003). A comparison of treatment-seeking pathological gamblers based on preferred gambling activity. Addiction, 98(5), 645-655. doi:10.1046/j.1360-0443.2003.00336.x

Popay, J., Roberts, H., Sowden, A., Petticrew, M., Arai, L., Rodgers, M., Britten, N., Roen, K., \& Duffy, S. (2006). Guidance on the conduct of narrative synthesis in systematic reviews. A product from the ESRC methods programme. Lancaster, UK: Lancaster University. Retrieved from http:// citeseerx.ist.psu.edu/viewdoc/download?doi=10.1.1.178.3100 $\&$ rep $=$ rep $1 \&$ type $=$ pdf

Siemens, J. C., \& Kopp, S. W. (2011). The influence of online gambling environments on self-control. Journal of Public Policy \& Marketing, 30(2), 279-293. doi:10.1509/jppm.30.2.279

Tremblay, N., Boutin, C., \& Ladouceur, R. (2008). Improved selfexclusion program: Preliminary results. Journal of Gambling Studies, 24(4), 505-518. doi:10.1007/s10899-008-9110-Z

Wardle, H., Moody, A., Griffiths, M., Orford, J., \& Volberg, R. (2011). Defining the online gambler and patterns of behavior integration: Evidence from the British Gambling Prevalence Survey 2010. International Gambling Studies, 11(3), 339-356. doi:10.1080/14459795.2011.628684 
Wood, R. T., \& Williams, R. J. (2009). Internet gambling: Prevalence, patterns, problems, and policy options. Guelph, ON: Ontario Problem Gambling Research Centre. Retrieved from http://www.jogoremoto.pt/docs/extra/XNFGmG.pdf
Wood, R. T., \& Williams, R. J. (2011). A comparative profile of the Internet gambler: Demographic characteristics, game-play patterns, and problem gambling status. New Media \& Society, 13(7), 1123-1141. doi:10.1177/1461444810397650 